\title{
Vipassana meditation practices enhance the parasympathetic activity during sleep: a case-control study of heart rate variability across sleep cycles
}

\begin{abstract}
Background: Sleep state related cardiac autonomic modulations are important for proper cardiovascular functions and to maintain homeostasis during sleep. The present study evaluates the efficacy of Vipassana meditation on cardio autonomic dynamicity across sleep cycles during sleep.

Methods: 26 Senior Vipassana meditation practitioners and 23 non-meditating control subjects participated in the study. Whole night polysomnography with lead II ECG were carried out using 32 channel, 2110 digital EEG system (Nihon Kohden, Japan) and with 16 channels Power Lab data acquisition system (AD Instruments, Australia. Differences in sleep variables across sleep cycles among the meditation practitioners and control groups (between groups) were analyzed by two way ANOVA with turkey posthoc test. The heart rate variability (HRV) was assessed during N2 and N3 and REM sleep state across three consecutive sleep cycles. The HRV variables-High frequency power (HF) and low frequency power (LF) density, their normalized units (HFnu and LFnu) and ratio (LF/HF ratio) were compared across sleep cycles using Analysis of variance with repeated measures for within group and ' $\mathrm{t}$ ' test for between groups.
\end{abstract}

Results: Vipassana meditators showed significantly high HFnu irrespective of sleep states across sleep cycles. During REM sleep, as HF nu was concomitantly enhanced along with increased LFnu, the LF/HF ratio was reduced in meditators. In addition, meditators showed distinct sleep architecture with enhanced slow wave sleep and REM sleep states along with significantly reduced intermittent awakenings indicative of proper sleep organisation.

Conclusion: Vipassana meditation practice helps to establish a proper cardio autonomic dynamicity during sleep with a persistent increase in parasympathetic activity across sleep states. Such a state of heightened parasympathetic activity especially during REM sleep helps to buffer the sympathetic surges and helps to maintain a proper sympatho-vagal balance essential for quality sleep and cardio protection.

Keywords: vipassana meditation, sleep cycles, heart rate variability

\author{
Volume 5 Issue 2 - 2017
}

\author{
Ravindra P Nagendra,' Sathiamma Sulekha,' \\ Arun Sasidharan,' Sathyaprabha TN,' \\ Nithyanda Pradhan, ${ }^{2}$ Raju TR,' Bindu M \\ Kutty' \\ 'Department of Neurophysiology, National Institute of Mental \\ Health and Neurosciences, India \\ 2Department of Psychopharmacology, National Institute of \\ Mental Health and Neurosciences, India
}

Correspondence: Bindu M Kutty, Department of Neurophysiology, National Institute of Mental Health and Neurosciences, Hosur Road, Bengaluru-560029, Karnataka, India, Tel 91802699 5I70,Email bindu.nimhans@gmail.com

Received: September II, 2016 | Published: January 19, 2017
Abbreviations: HRV, heart rate variability; LF, low frequency; $\mathrm{HF}$, high frequency; LFnu, normalised if frequency; HFNu, normalised hf frequency; N2, sleep stage 2; N3, sleep stage 3; SWS, slow wave sleep; REM, rapid eye movement; NREM, non rapid eye movement; NN, r to $r$ wave interval in ecg; SDNN, standard deviation of nn interval; RMSSD, root mean square of sdnn; NN50, nn interval more than $50 \mathrm{~m} \mathrm{sec}$; PNN50, percentage of nn50

\section{Introduction}

Sleep is an important behavioural state essential for health and survival, refreshes and restores all the bodily functions. Human sleep architecture has a stable morphology with alternating NREM and REM sleep states reflecting the dynamicity of brain activities including the homeostatic and circadian regulatory mechanisms. Cardiac autonomic activity during sleep is highly dynamic and varies according to sleep states. Heart rate variability (HRV) is anon invasive and reliable measure of cardiac autonomic activity during sleep. ${ }^{1}$ During slow wave sleep (SWS), the HRV shows increased power in high frequency (HF) range and its normalized units (HFnu) indicating the predominance of parasympathetic activity. Whereas, during REM sleep, HRV shows predominance of power in the low frequency range (LF) and its normalized units (LFnu), indicating greater sympathetic activity. Thus, cardiac autonomic activity switches from vagally predominant SWS to sympathetically predominant REM sleep in every sleep cycle. ${ }^{2-4}$ Such sleep state dependent alteration in autonomic activity is considered as one of the mechanisms for regulating the sleep-wake cycle and is a prerequisite for maintaining homeostasis during sleep. ${ }^{5}$ During aging, such precise sleep state dependent cardio autonomic dynamicity is compromised leading to an attenuation of parasympathetic activity ${ }^{6}$ along with an overall increase in sympathetic activity and overt sympathetic surges during REM sleep. ${ }^{7}$ Such REM sleep associated sympathetic surges is reported to be a risk factor for unfavourable cardiac events. ${ }^{8}$ Therefore effective buffering of sympathetic surges during sleep is crucial for establishing quality sleep and cardiac health.

Emerging evidences suggest that psycophysiological practices like meditation help to maintain sympatho-vagal balance by increasing the vagal tone. ${ }^{9}$ Meditation practices bring down the heart rate, systolic blood pressure, and total peripheral resistance ${ }^{10}$ and decreases the LFnu and LF/HF ratio, thus favouring sympatho-vagal balance towards parasympathetic predominance. ${ }^{11}$ Imaging studies have shown that mindfulness meditation practices activate the neural structures associated with autonomic nervous system especially the anterior cingulate cortices, whose activity is highly correlated with 
high frequency variations in HRV. ${ }^{12}$ Thus, meditative practices are beneficial to establish the cardiovascular efficacy by effectively modulating the cardiac autonomic activity. However, beneficial effect of meditation practices on cardiac autonomic activity during sleep has not been studied effectively. Cyclic meditation and Taiwanese practices are reported to shift the cardiac autonomic balance towards parasympathetic dominance during sleep. ${ }^{13}$ As sleep follows an alternating NREM-REM sleep events with sleep state dependent cardio autonomic modulations, how meditative practices help to regulate such sleep cycle dependent cardio autonomic dynamicity is still not understood. We have studied the changes in sleep architecture among Vipassana mediation practitioners. ${ }^{14,15}$ The present study evaluated the HRV across sleep cycles in long term practitioners of Vipassana meditation. This would help us to elucidate how meditative practices modulate the cardiac autonomic activity across sleep cycles.

\section{Material and methods}

\section{Participants}

A total of 49 healthy male subjects between 30-60years of age participated in the study. They belong to two groups Vipassana Meditation practitioners $(n=26)$ and control groups $(n=23)$.

Vipassana Meditators are senior practitioners with meditation practice 2-4hours/day for more than 3years and have undergone many advanced courses in Vipassana meditation. The Vipassana meditators were recruited through the Vipassana Research Institute at Igathpuri, India, the world head quarters for Vipassana meditation. All of them were employed and with family responsibilities, none of them were monks nor stayed in any monastery. Vipassana meditation is one of the most ancient forms of meditative technique said to have taught and practiced by the Buddha around 2500years. Vipassana meditation involves the techniques of mindfulness that follows the tradition of Sayagyi U Ba Khin as taught by S.N. Goenka. Vipassana meditation practice involves the strategy of mindfulness wherein, the meditators learns to notice and witness the perceptions of the senses and the thoughts arising in the mind without reacting to them, like an on lookers, and to focus their attention on their bodily activities in their true perspective, in their true nature with an equanemous and non reactive mind. The controls were healthy volunteers who have not practiced any form of meditation/yoga or any other mind-body techniques. They were enrolled for the study through local advertisements and contacts. The study was initiated after obtaining approval from the Institute Human Ethics Committee (IEC No.RPA/058/05). The participants were detailed about the objectives of the study and a written informed consent was taken from them prior to the study participants from both the groups were matched for demographic characteristics such as age, socioeconomic and educational status.

Before recruitment, the participants were assessed for their routine sleep habits and were examined for medical, psychiatric, neurological and other disease conditions. Participants were requested to maintain a sleep $\log$ (UCLA sleep log) for a week prior to the study. The total sleep duration of both controls and meditators was about 6.5 to 7 hours (between 10:30-11:30 P.M. to 5-6A.M). The Subjects who were on regular medications or regular tobacco or alcohol consumption or those reported to have acute or chronic sleep disturbance/restriction/ deprivation, and if found to have any signs and symptoms of any disease conditions were excluded from the study. The selected participants $(n=49)$ underwent whole night polysomnography in the Sleep Laboratory, Department of Neurophysiology, NIMHANS for two consecutive nights.

\section{Polysomnography}

Whole night Polysomnographic recordings with lead II ECG were carried out for two consecutive nights in a semi sound-proof air conditioned sleep cabin during subjects' habitual sleep time. Polysomnography was recorded by using 32 channel digital EEG system with a sampling rate of $512 \mathrm{~Hz}$ (EEG-2110, Nihon Kohden Corporation, Japan) simultaneously with ECG in lead II by 16 channel Power Lab data acquisition system with a sampling rate $1024 \mathrm{~Hz}$ (AD instruments, Australia). The lights were switched off during sleep until the subject woke up by themselves in the morning. The first night sleep was recorded as an adaptation to the laboratory environment and the second night's polysomnographic recording was used for sleep scoring and HRV analysis. The offline scoring of sleep was carried out according to the American Society of sleep medicine (AASM) guidelines ${ }^{16}$ independently by two trained scorers with more than five years of experience in sleep stage scoring and were blind to the details of the subjects.

\section{Heart rate variability}

In order to assess the heart rate variability (HRV), 5minutes of artefact-free electrocardiogram (ECG) recordings were selected from N2, N3 and REM sleep states in the first three sleep cycle. The N1 sleep state being too short a period was not considered for HRV analysis. ECG records with high noise, respiratory and movement artefacts, frequent ectopics, etc., were rejected. The selected ECG epochs were further scrutinised for ectopic or noise, and was interpolated with R-R interval. HRV was obtained as per guidelines of Taskforce report 1996.

Power spectral analysis of RR interval was carried with Fast Fourier transformation based on non parametric algorithm using Welch window using inbuilt HRV software of AD instrument. Power spectral densities of low frequency (LF: $0.04-0.15 \mathrm{~Hz}$ ), high frequency (HF: $0.15-0.50 \mathrm{~Hz}$ ), their normalised units (LFnu and HFnu) and their ratio (LF/HF) during N2, N3 and REM sleep across three sleep cycles were compared within and between the two groups. Within group analysis was carried out to ascertain how HRV measures varies with different sleep states across sleep cycles in each group (within controls and meditators) Between groups analysis was carried to find out the differences in the HRV modulation during different sleep stages across sleep cycles in meditators when compared to controls.

\section{Statistical methods}

All statistical analyses were carried out using SPSS/PASW Version 18 software (SPSS Ltd, Hong Kong). Interrater reliability of Sleep stage scoring by two independent scorers was done using kappa statistics. Sleep stage comparison between two groups was carried using ANOVA. Within group difference in time domain and Power spectral densities of HRV during different sleep stages across sleep cycles was analyzed using analysis of variance with repeated measures. ${ }^{17}$ Mauchly's test was applied to test the assumption of sphericity. Green House Gessier corrections were applied for those HRV variables violated sphericity assumptions. Main effect of group and sleep stages was carried out. For multiple comparisons between various sleep states across sleep cycles Bonferroni posthoc test was applied for HRV parameters $p<0.05$ was considered to be significant. Effect size was estimated to assess the effect of meditation on HRV. ' $t$ ' test was applied to compare HRV between controls and meditators to ascertain the differences of HRV during each sleep states in all the sleep cycles. $\mathrm{p}<0.016$ (after Bonferroni correction) as significant level. 


\section{Results}

Both controls and Vipassana meditators were matched for their age (controls-45.18 \pm 9.33 years and meditators- $45.79 \pm 10.32$ years, $\mathrm{F}=(1,48), 1.09, \mathrm{p}=0.29)$, socio-economic and educational status. All subjects reported to have good sleep with the sleep efficiency index more than $90 \%$. Sleep stages across sleep cycles was assessed in 49 subjects (controls $=23$, Vipassana meditators $=26$ ) and HRV during $\mathrm{N} 2, \mathrm{~N} 3$ and REM sleep stages across sleep cycles was estimated in 22 subjects controls $=9$, meditators $=13$.

\section{Whole night sleep architecture and sleep variables}

Cohens kappa Inter rater reliability of sleep scoring of two independent scorers was 0.85 demonstrating that the sleep stage scoring was highly satisfactory. The total sleep time (TST) controls-365.35 \pm 57.54 minutes, meditators$380.85 \pm 64.54$ minutes, $\mathrm{F}=(1,48)=0.90, \quad \mathrm{p}=0.341$, sleep efficiency index (\%) Controls-91.81 \pm 3.80 , meditators- $93.63 \pm 3.62, \mathrm{~F}=(1,48)=$ $3.40, \mathrm{p}=0.071$ and wake after sleep onset (WASO) controls$60.28 \pm 8.25$ minutes, meditators $-46.82 \pm 5.58$ minutes, $\mathrm{F}=(1,48)=1.89$, $\mathrm{p}=0.17$ were comparable between meditators and controls. Table 1 provides the details of sleep variables studied across the night between controls and meditators. Meditators showed significantly less duration of $\mathrm{N} 1 \mathrm{~F}=(1,48)=4.56, \mathrm{p}=0.037$ and $\mathrm{N} 2 \mathrm{~F}=(1,48)=10.49, \mathrm{p}=0.002$, whereas, the duration of $\mathrm{N} 3 \mathrm{~F}=(1,48)=7.15, \mathrm{p}=0.010$ and REM sleep $\mathrm{F}=(1,48)=18.16, \mathrm{p}=0.000$ was significantly increased in Vipassana meditators when compared to controls.

\section{Sleep variables across sleep cycles}

Table 1 provides the details of sleep stages across sleep cycles between controls and meditators. Among the meditation practitioners when compared to controls, N2 state showed significant reduction in the first sleep cycle, whereas, it was comparable in the consecutive sleep cycles. first sleep cycle-N2 percentage- $\mathrm{F}=(1,48)=7.23, \mathrm{p}=0.010, \mathrm{~N} 2$ duration- $\mathrm{F}=(1,48)=7.08, \mathrm{p}=0.011$; second sleep cycle-N2 percentage $\mathrm{F}=(1,48)=3.78, \mathrm{p}=0.058, \mathrm{~N} 2$ duration $\mathrm{F}=(1,48)=2.00, \mathrm{p}=0.16$; Third sleep cycle $-\mathrm{N} 2$ percentage $\mathrm{F}=(1,48)=2.78, \mathrm{p}=0.078$, N2 duration $\mathrm{F}=(1,48)=2.20, \mathrm{p}=0.14$. The duration and percentage of sleep stage N3 was significantly high in the second and third sleep cycle in meditators (Figure 1) first sleep cycle-N3 percentage- $\mathrm{F}=(1,48)=2.52$, $\mathrm{p}=0.11, \mathrm{~N} 3$ duration- $\mathrm{F}=(1,48)=0.89, \mathrm{p}=0.34$; second sleep cycle-N3 percentage- $\mathrm{F}=(1,48)=3.26, \mathrm{p}=0.05, \mathrm{~N} 3$ duration $-\mathrm{F}=(1,48)=5.31$, $\mathrm{p}=0.02$; third sleep cycle $=\mathrm{N} 3$ percentage $\mathrm{F}=(1,48)=8.67$, $\mathrm{p}=0.005, \mathrm{~N} 3$ duration $\mathrm{F}=(1,48)=5.07, \mathrm{p}=0.029$. Meditators showed significant reduction in the REM sleep onset latency (ROL in minutes) when compared to controls $\mathrm{F}=(1,48)=9.71 \mathrm{p}=0.003$ The REM sleep was significantly high in the third sleep cycle (Figure 2) first sleep cycle - REM sleep percentage- $\mathrm{F}=(1,48)=1.71$, $\mathrm{p}=0.19$, REM sleep duration- $\mathrm{F}=(1,48)=0.15, \mathrm{p}=0.69$; second sleep cycle- REM sleep percentage- $\mathrm{F}=(1,48)=2.49, \mathrm{p}=0.12$, REM sleep duration- $\mathrm{F}=(1,48)=1.14, \mathrm{p}=0.29$; third sleep cycle- $\mathrm{REM}$ sleep percentage- $F=(1,48)=8.07, p=0.007$, REM sleep duration $\mathrm{F}=(1,48)=8.07, \mathrm{p}=0.007$. The representative hypnogram of meditator and control is shown in Figure 3.

In summary, the sleep states dynamics across sleep cycles were different among Vipassana meditators with reduced N2, progressive increase in N3 and REM sleep states in the subsequent sleep cycles.

\section{HRV assessment}

The details of time and frequency domain parameters of HRV during N2, N3 and REM sleep states across three sleep cycles, group and sleep stage effect, , effect size of meditation on HRV parameters are provided in Tables $2 \& 3$. Figure 4 provides the details of HRV, for low and high frequency power, their normalized units and ratio during N2, N3 and REM sleep states across sleep cycles.

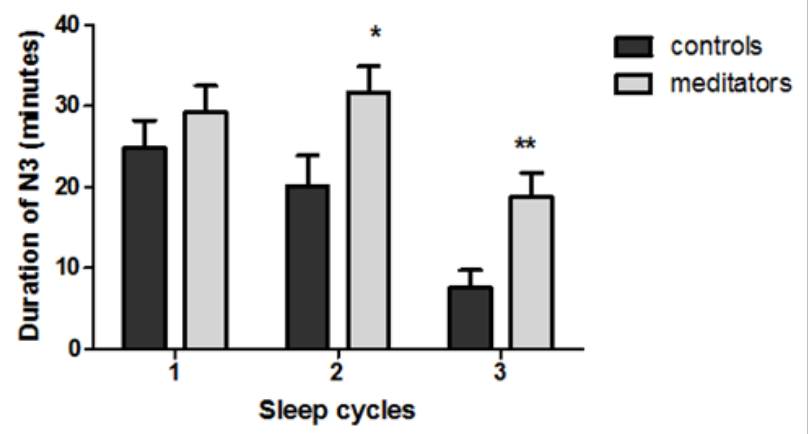

Figure I Shows that duration of $\mathrm{N} 3$ is significantly high in meditators during second and third sleep cycle when compared to controls. ${ }^{*} p<0.05,{ }^{* *} p<0.001$.

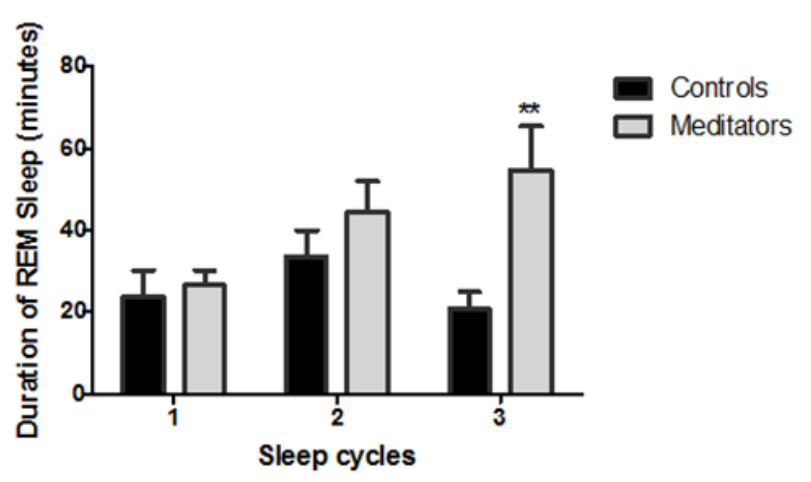

Figure 2 Shows that duration of REM sleep is significantly higher in meditators when compared to controls during third sleep cycle. ${ }^{* *} p<0.001$.

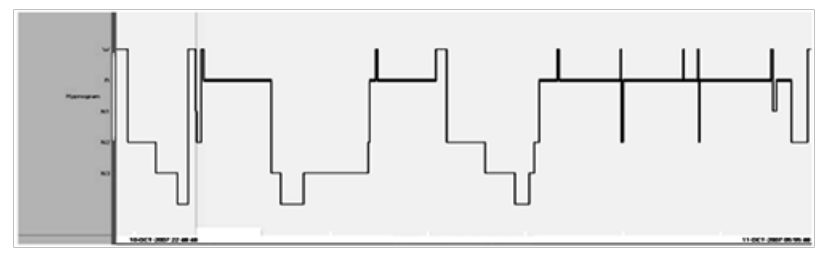

Meditator (45 years)

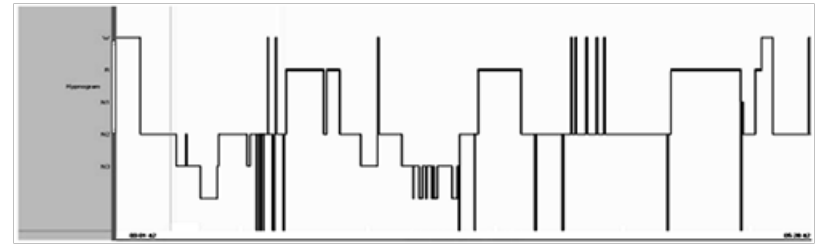

Figure 3 Shows representative hypnograpm of a meditator and control of 45 years of age. Meditator shows increase in N3 and REM sleep stage with less intermittent awakenings than that of controls.

\section{HRV dynamics during sleep cycles}

Repeated measures of analysis of variance of time domain parameters of HRV (Table 2) showed no group or sleep stage effect. However, ' $\mathrm{t}$ ' test for nn50 and pnn50 showed a higher value during REM sleep among meditators when compared to controls. Repeated measures of analysis of variance of frequency domain parameters 
of HRV (Table 3) showed a significant group and sleep stage effect for normalised units of high frequency (HFnu) and low frequency (LFnu) and ratio (LF/HF) in all the three sleep cycles. HRV during various sleep stages across three sleep cycles in both controls and meditators showed sleep state dependent modulations (Figure 4). HFnu an indicator of parasympathetic activity, showed a progressive increase from N2 state to N3 sleep state $(\mathrm{p}=0.002)$ and subsequently a significant reduction during REM sleep $(\mathrm{p}=0.00)$ across each sleep cycles in each group. In contrast, both LF power $(\mathrm{p}=0.016)$ and LFnu $(\mathrm{p}=0.029)$ indicators of sympathetic activity, showed a progressive decrease from $\mathrm{N} 2$ to $\mathrm{N} 3$ sleep state but increased during REM sleep (LF nu- $p=0.000$ ). Hence, the LF/HF ratio was reduced during N3 sleep state $(\mathrm{p}=0.025)$ but increased during REM sleep $(\mathrm{p}=0.003)$.

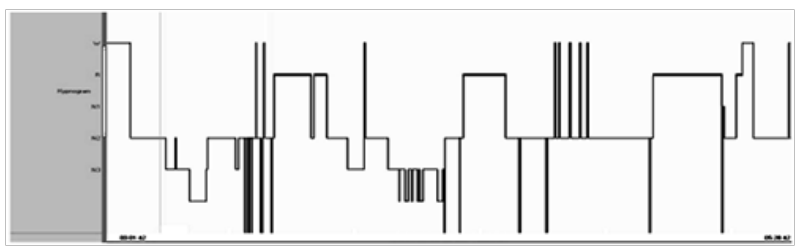

Figure $4 * \mathrm{p}<0.016$ after Bonferroni corrections for ' $\mathrm{t}$ ' test comparing HRV variables between controls and meditators during N2,N3 and REM sleep stages across three sleep cycles.

The normalised units of HRV between meditators and control in each sleep state across sleep cycles was compared with ' $t$ ' test (Figure 4). Vipassana meditators showed increase in HFnu during N2 (first sleep cycle $-\mathrm{t}=-4.82, \mathrm{p}=0.00$, second sleep cycle $-\mathrm{t}=-2.94, \mathrm{p}=0.007$ ), $\mathrm{N} 3$ (second sleep cycle $-\mathrm{t}=-4.43, \mathrm{p}=0.000$; third sleep cycle $-\mathrm{t}=-2.45$, $\mathrm{p}=0.003$ ) and REM sleep states (first sleep cycle- $\mathrm{t}=-3.00, \mathrm{p}=0.005$; second sleep cycle- $t=-2.19, p=0.0040$; third sleep cycle- $t=-2.54$, $\mathrm{p}=0.002)$ than controls. Meditators showed significantly less LF nu during N2 (first sleep cycle- $=3.14, p=0.003$; second sleep cycle- $t$ $=2.25, \mathrm{p}=0.003$ ) and $\mathrm{N} 3$ sleep states (second sleep cycle- $\mathrm{t}=-2.94$, $\mathrm{p}=0.009$, third sleep cycle $-\mathrm{t}=1.46, \mathrm{p}=0.17$ ) than controls, but, during REM, LFnu was comparable between meditators and controls (first sleep cycle $-\mathrm{t}=0.69, \mathrm{p}=0.49$; second sleep cycle- $\mathrm{t}=0.83$, $\mathrm{p}=0.41$; third sleep cycle- $\mathrm{t}=1.18, \mathrm{p}=0.24)$. The $\mathrm{LF} / \mathrm{HF}$ ratio was significantly less in meditators throughout the sleep states $(\mathrm{N} 2$ - first sleep cycle $\mathrm{t}=4.08$, $\mathrm{p}=0.00$; second sleep cycle $\mathrm{t}=1.15, \mathrm{p}=0.26$; $\mathrm{N} 3$ - third sleep cycle $\mathrm{t}=2.37, \mathrm{p}=0.003$; REM - first sleep cycle $-\mathrm{t}=2.53, \mathrm{p}=0.002$; second sleep cycle $-\mathrm{t}=2.23, \mathrm{p}=0.0042$; third sleep cycle $\mathrm{t}=2.29, \mathrm{p}=0.004$ ) as the HF nu was high across sleep states.

In brief, the study showed differences in HRV measures during sleep between Vipassana meditators and controls. The meditators showed significant increase in the parasympathetic activity (HFnu) in all the sleep states i.e N2, N3 and REM, and significantly reduced sympathetic activity (LFnu) in N2 and N3. But, the sympathetic activity during REM sleep was comparable between meditators and controls. Therefore, inspite of comparable sympathetic activity during REM sleep, enhanced parasympathetic activity among meditators has contributed to significantly less LF/HF ratio indicating effective buffering of REM associated sympathetic activity. LF/HF ratio even in N2 and N3 was also significantly less in meditators, thus, depicting the predominance of parasympathetic activity among meditators across all the sleep states.

\section{Discussion}

We have demonstrated the changes in cardiac autonomic modulations during sleep among Vipassana meditation practitioners. The vipassana meditators showed distinct changes in HRV such as enhanced HFnu throughout sleep states indicating predominance of parasympathetic activity and reduced $\mathrm{LF} / \mathrm{HF}$ ratio implying establishment of proper sympatho vagal balance. The control subjects showed an increase in LFnu and LF/HF ratio across sleep states suggestive of sympathetic predominance during sleep irrespective of the sleep states.

In the present study, both controls and meditators showed sleep state dependent modulations in cardiac autonomic activity, with higher HFnu during N3 and higher LFnu during REM sleep state; suggestive of enhanced parasympathetic and sympathetic activity respectively during N3 and REM sleep states. Among HRV variables, the normalized units of high (HFnu) and low (LFnu) frequency power reflect the cardiac autonomic dynamicity in sleep and represent a controlled and balanced behavior of parasympathetic and sympathetic limb of autonomic nervous system. ${ }^{1}$ High frequency oscillations and their normalized units (HFnu) in HRV are considered as a marker of parasympathetic activity. ${ }^{18}$ As the sleep deepens from lighter to deeper states, the heart rate and blood pressure reduces and rhythmic respiration brings about an increase in high frequency power of HRV indicative of parasympathetic activity. During REM sleep, the heart rate and blood pressure increases and the respiratory patterns become irregular, leading to an increased low frequency power of HRV due to increase in sympathetic activity. ${ }^{19}$ Such cardiac autonomic modulations are related to the altered metabolic status associated with different sleep states, wherein, the metabolic demands become more during REM and less during slow wave sleep states..$^{20}$ Though both meditators and controls showed sleep state dependent changes in cardiac autonomic modulations, Vipassana meditators, across all the sleep states, exhibited higher parasympathetic activity, thereby reducing LF/HF ratio indicative of proper Sympathovagal balance. This cardio autonomic dynamicity associated with meditation practice is more evident during REM sleep states where we have observed a concomitant increase in parasympathetic activity along with sympathetic activity.

Many randomized controlled studies have shown the positive regulatory role of Yoga/Meditation interventions help to increase the muscular strength and dexterity; reduce the behavioural signs of anxiety, depression and enhancement of parasympathetic activity. ${ }^{21-24}$ Increased internalised attention during meditation is known to bring about synchronisation between central and peripheral autonomic networks, modulation of attention and emotional resilience, establishes a hypo metabolic state with parasympathetic dominance ${ }^{25,26}$ thus, bringing a sense of deep relaxation. ${ }^{27,28}$ Cyclic meditation practices are shown to enhance the subjective sense of quality sleep with increased vagal activity. ${ }^{13}$ We have reported distinct changes in sleep architecture among senior Vipassana meditation practitioners with enhanced slow wave sleep, REM sleep states even among elderly meditators. ${ }^{14,15}$ Thus, our study showed that Vipassana meditation practice help to defy the age related changes in sleep quality and structure. Such meditation induced enhancement of slow wave sleep and REM sleep states along with enhanced alpha activity has been reported among senior practitioners of Transcendental Meditation. ${ }^{29}$ These meditation induced changes in sleep organisation has been considered as the result of increased plasticity changes and efficient thalamocortical oscillations and structural changes such as enhanced cortical thickness of dorsal anterior cingulategyrus. ${ }^{30}$ These plasticity changes probably could have helped in attenuating the effect of sleep deprivation on cognitive impairment ${ }^{31}$

In the present study, in addition to changes in sleep architecture such as enhanced N3 and REM sleep states, we have also 
observed changes in cardio autonomic modulations with enhanced parasympathetic activity in both of these sleep stages. Imaging studies have demonstrated increased activity in the regions of left insula, subgenual and anterior cingulate during meditation practice which are the part of central autonomic network that brings about parasympathetic activity. ${ }^{11,12}$ There are evidences demonstrating that the areas that were predominantly active during waking hours get reactivated during sleep, especially during REM sleep ${ }^{32}$. Therefore, it could be hypothesized that Vipasana meditation practices would have accentuated the activation of parasympathetic activity during sleep among meditators, thus, enhancing the parasympathetic activity during sleep, including that of REM sleep state. Control subjects did not show such persistent enhancement of parasympathetic activity especially during REM sleep.

Both controls and meditators, showed predominance of sympathetic activity (LFnu) during REM sleep. However, in meditators, in addition to enhanced sympathetic activity, the parasympathetic activity (HF nu) was significantly enhanced during REM sleep. Control subjects did not show such concomitant increase in parasympathetic activity associated with REM sleep. The HF nu was about $59 \%$ of LFnu in controls, but, in meditators, the HF nu was about $104 \%$ of that of LF nu highlighting the concomitant increase in parasympathetic activity among meditators. This variations in sympathetic and parasympathetic activity during REM sleep lead to an increase of LF/ HF ratio among controls as reported by others..$^{33}$ During REM sleep, as the parasympathetic activity was high in meditators the LF/ HF ratio was significantly reduced. Thus, highlight that meditatiors could effectively buffer the sympathetic activity during REM sleep and the establishes a proper sympathovagal balance. Such effective buffering of sympathetic activity was not observed in controls thereby brining the autonomic balance more towards sympathetic predominance. Sympathetic activity was high among controls across the sleep states which could be considered as a mark of normal aging process that is associated with reduced parasympathetic functions with a concomitant enhancement of sympathetic functions. ${ }^{7}$ Aging is associated with such poorly controlled cardiovascular variability and with reduced cardiovascular capability to respond effectively to the stress and perturbations during REM sleep. ${ }^{8}$ Reports suggest that almost $40 \%$ higher incidences of myocardial infarction, stroke, sudden cardiac death, conduction blocks etc occur during REM sleep. ${ }^{8,34}$ Central sympathetic inhibition with pharmacological agents is shown to reestablish the sleep quality and autonomic modulations. ${ }^{35}$ Therefore, effective buffering of sympathetic surges during REM sleep is one of the prerequisite towards ensuring cardio protection and quality sleep. The effective buffering of sympathetic surges during sleep is dependent on bar reflex sensitivity which is known to increase during REM sleep in the successive sleep cycles. ${ }^{36}$ Meditation practices ${ }^{37}$ and melatonin administration ${ }^{38}$ help to enhance the sensitivity of baroreflex mechanism. Meditation practices in turn enhance the melatonin levels..$^{39,40}$ We have also observed enhanced levels of both morning and evening levels of plasma melatonin among senior Vipassana meditation practitioners..$^{41}$ Thus meditative practices help in effective buffering of sympathetic surges during REM sleep. This is one of the fewer studies highlighting the effectiveness of meditative practices on effective cardio autonomic modulations during sleep. This observation is of paramount importance in considering meditative practices in general and Vipassana meditation practices in particular as an ideal approach to re instate enhanced parasympathetic activity during sleep especially in present time wherein we are beginning to understand the existence of various sleep related problems and other co morbidities as a part of change in life style.

\section{Conclusion}

In conclusion, Vipassana meditation practices lead to proper cardio autonomic modulations during sleep. Proficient practice of Vipassana meditation helps to increase the parasympathetic activity throughout sleep and effectively buffers the sympathetic surges during REM sleep. The present study has limitations as we have not studied the other variables such as the rate and depth of respiration, variations in body temperature etc all of which affect the HRV during sleep. Additionally, a simultaneous EEG/fMRI acquisition of HRV during sleep or direct assessment of baroreflex mechanisms etc would have provided additional details of the HRV dynamicity associated with meditation practices. Like in earlier studies, the findings of our study support the meditation induced enhancement of vagal tone including during sleep and neurophysiologic growth. ${ }^{21,42}$

\section{Acknowledgments}

The study was funded by CCRYN (Central Council for Research in Yoga and Naturopathy), Dept of AYUSH, Ministry of Health and Family welfare Govt of India, New Delhi (project no. 12-2/ Res / CCRYN/ 2003-2004). A special note of our gratitude to the Vipassana Research Institute, Igatpuri, India for their constatnt support and encouragement. . We also thank all the participants for their support and co-operation to make the study possible. We greatly acknowledge the technical help provided by Mr. M Ramachandra, Mr. B G Nagaraj towards the completion of the study. We also thank Dr. Mariamma Philip, Department of Bio Statitistics, NIMHANS for expert opinion on statistical methods used in the study. Our heartfelt thanks are due to NIMHANS for the constant support and encouragement throughout the study.

\section{Conflicts of interest}

Author declares there are no conflicts of interest.

\section{Funding}

None.

\section{References}

1. Burr RL. Interpretation of normalized spectral heart rate variability indices in sleep research: a critical review. Sleep. 2007;30(7):913-919.

2. Otzenberger H, Simon C, Gronfier C, et al. Temporal relationship between dynamic heart rate variability and electroencephalographic activity during sleep in man. Neurosci Lett. 1997;229(3):173-176.

3. Pedemonte M, Rodriguez-AlvezA, Velluti, RA. Electroencephalographic frequencies associated with heart changes in RR interval variability during paradoxical sleep. Auton Neurosci. 2005;123(2):82-86.

4. Trinder J, Kleiman J, Carrington M, et al. Autonomic activity during human sleep as a function of time and sleep stage. J Sleep Res. 2001;10(4):253-264.

5. Cortelli P, Lombardi C, Montagna P, et al. Baroreflex modulation during sleep and in obstructive sleep apnea syndrome. Auton Neurosci. 2012;169(1):7-11.

6. Jurysta F, van de Borne P, Lanquart JP, et al. Progressive aging does not alter the interaction between autonomic cardiac activity and delta EEG power. Clin Neurophysiol. 2005;116(4):871-877.

7. Brandenberger G, Viola AU, Ehrhart J, et al. Age-related changes in cardiac autonomic control during sleep. J Sleep Res. 2003;12(3):173180 . 
8. Viola AU, Tobaldini E, Chellappa SL, et al. Short-term complexity of cardiac autonomic control during sleep: REM as a potential risk factor for cardiovascular system in aging. PLoS One. 2011;6(4):e19002.

9. Nesvold A, Fagerland MW, Davanger S, et al. Increased heart rate variability during nondirective meditation. Eur J Prev Cardiol. 2012;19(4):773-780.

10. Barnes VA, Davis HC, Murzynowski JB, et al. Impact of meditation on resting and ambulatory blood pressure and heart rate in youth. Psychosom Med. 2004;66(6):909-914.

11. Wu SD, Lo PC. Inward-attention meditation increases parasympathetic activity: a study base on heart rate variability. Biomed Res. 2008;29(5):245-250.

12. Tang YY, Ma Y, Fan Y, et al. Central and autonomic nervous system interaction is altered by short-term meditation. Proc Natl Acad Sci U S A.2009; 106(22): 8865-8870.

13. Patra $\mathrm{S}$, Telles $\mathrm{S}$. Heart rate variability during sleep following the practice of cyclic meditation and supine rest. Appl Psychophysiol Biofeedback. 2010;35(2):135-140.

14. Pattanashetty R, Sulekha S, Pradhan N, et al. Practitioners of Vipassana meditation exhibit enhanced slow wave sleep and REM sleep states across different age groups. Sleep and Biological Rhythms. 2010;8(1): 34-41.

15. Sulekha S, Thennarasu K, Raju TR, et al. Evaluation of sleep architecture in practitioners of SudharshanKriya yoga and Vipassana meditation. Sleep and Biological Rhythms. 2006;4(3):207-214.

16. Silber $\mathrm{MH}$, Ancoli-Israel S, Bonnet $\mathrm{MH}$, et al. The visual scoring of sleep in adults. J Clin Sleep Med. 2007;3(2):121-131.

17. Younsuk Lee. What repeated measures analysis of variances really tells us. Korean J Anesthesiol. 2015;68(4):340-345.

18. Berntson GG, Bigger JT, Eckberg DL, et al. Heart rate variability: origins, methods, and interpretive caveats. Psychophysiology. 1997;34(6):623-648.

19. Elsenbruch S, Harnish MJ, Orr WC. Heart rate variability during waking and sleep in healthy males and females. Sleep. 1999;22(8):1067-1071.

20. Wilde-Frenz J, Schulz H. Rate and distribution of body movements during sleep in humans. Percept Mot Skills. 1983;56(1):275-283.

21. van der Zwan JE, de Vente W, Huizink AC, et al. Physical Activity, Mindfulness Meditation, or Heart Rate Variability Biofeedback for Stress Reduction: A Randomized Controlled Trial. Appl Psychophysiol Biofeedback. 2015;40(4):257-268.

22. Lin SL, Huang CY, Shiu SP, et al. Effects of Yoga on Stress, Stress Adaption, and Heart Rate Variability Among Mental Health Professionals--A Randomized Controlled Trial. Worldviews Evid Based Nurs. 2015;12(4):236-245.

23. Sawane MV, Gupta SS. Resting heart rate variability after yogic training and swimming: A prospective randomized comparative trial. Int $J$ Yoga. 2015;8(2):96-102.

24. Lau C, Yu R, Woo J, et al. Effects of 12 Week Hatha Yoga Intervention on Cardiorespiratory Endurance, Muscular Strength and Enduranceand Flexibility in Hong Kong Chinese Adults: A Controlled Clinical Trial. Evid Based Complement Alternat Med. 2015;8:1-12.

25. Murata T, Takahashi T, Hamada T, et al. Individual trait anxiety levels characterizing the properties of zen meditation. Neuropsychobiology. 2004;50(2):189-194.
26. Young JD, Taylor E. Meditation as a Voluntary Hypometabolic State of Biological Estivation. News Physiol Sci. 1998;13:149-153.

27. Telles S, Raghavendra BR, Naveen KV, et al. Changes in autonomic variables following two meditative states described in yoga texts. $J$ Altern Complement Med. 2013;19(1):35-42.

28. Krygier JR, Heathers JA, Shahrestani S, et al. Mindfulness meditation, well-being, and heart rate variability: a preliminary investigation into the impact of intensive Vipassana meditation. Int $J$ Psychophysiol. 2013;89(3):305-313.

29. Mason L, Alexander CN, Travis FT, et al. Electrophysiological correlates of higher states of consciousness during sleep in long-termpractitioners of the Transcendental Meditation program. Sleep. 2013;20(2):102-110.

30. Hölzel BK, Ott U, Gard T, et al. Investigation ofmindfulnes meditation practitioners with voxel-based morphometry. Soc Cogn Affect Neurosci. 2008;3(1):55-61.

31. Chatterjee A, Ray K, Panjwani U, ET AL. Meditation as an intervention for cognitive disturbances following total sleep deprivation. Indian $J$ Med Res. 2012;136(6):1031-1038.

32. Maquet $\mathrm{P}$, Laureys S, Peigneux $\mathrm{P}$, et al. Experience-dependent changes in cerebral activation during human REM sleep. Nat Neurosci. 2000;3(8):831-836.

33. Cabiddu R, Cerutti S, Viardot G, ET AL. Modulation of the SympathoVagal Balance during Sleep: Frequency Domain Study of Heart Rate Variability and Respiration. Front Physiol. 2012;3:45.

34. Verrier RL, Muller JE, Hobson JA. Sleep, dreams, and sudden death: the case for sleep as an autonomic stress test for the heart. Cardiovasc Res. 1996;31(2):181-211.

35. Yamazaki T, Asanoi H, Ueno H, et al. Central sympathetic inhibition augments sleep-related ultradian rhythm of parasympathetic tone in patients with chronic heart failure. Circ J. 2005;69(9):1052-1056.

36. Legramante JM, Marciani MG, Placidi F, et al. Sleep-related changes in baroreflex sensitivity and cardiovascular autonomic modulation. $J$ Hypertens. 2003;21(8):1555-1561.

37. Sivasankaran S, Pollard-Quintner S, Sachdeva R, et al. The effect of a six week program of yoga and meditation on brachial artery reactivity: do psychosocial interventions affect vascular tone? Clin Cardiol. 2006;29(9):393-398.

38. Girouard H, Denault C, Chulak C, ET AL. Treatment by n-acetylcysteine and melatonin increases cardiac baroreflex and improves antioxidant reserve. Am J Hypertens. 2004;17(10):947-954.

39. Harinath K, Malhotra AS, Pal K, et al. Effects of Hatha yoga and Omkar meditation on cardiorespiratory performance, psychologic profile, and melatonin secretion. J Altern Complement Med. 2004;10(2):261-268.

40. Tooley, GA, Armstrong, SM, et al. Acute increases in night-time plasma melatonin levels following a period of meditation. Biol Psychol. 2000;53(1):69-78

41. Nagendra RP, Maruthai, N, Kutty BM. Meditation and its regulatory role on sleep. Front Neurol. 2012;3:54.

42. Heart rate variability: standards of measurement, physiological interpretation and clinical use. Task Force of the European Society of Cardiology and the North American Society of Pacing and Electrophysiology. European Heart Journal. 1996;93(5):1043-1068. 